\title{
CAPILLARY POISONS AND ACIDOSIS *
}

\author{
GEORGE B. WALlACE, M.D., ANd EMIL J. PELlini, M.D. \\ NEW YORK
}

\section{INTRODUCTION}

In his admirable review of the functional activity of the capillaries and venules, Hooker ${ }^{1}$ brings out the facts that these structures change in caliber independently of the larger vessels, that they function actively and are responsive to both chemical and nervous influences. $\mathrm{He}$ pictures a nervous control, maintaining a tone of the capillary beds over the body as a whole, and a chemical regulation, usually local in character and effective according to the passing need of particular tissues.

As Hooker points out the story of capillary function is far from complete. What facts are known have to do with contraction and dilatation, and the question of capillary permeability is practically untouched. In an ultimate analysis, the function of the capillaries is to allow of the passage of oxygen and nutriment from the blood to tissues and the removal of waste products from tissues to the blood. A physiologic permeability is essential, therefore, for the nutrition and activity of tissues, and the complete mechanism of dilatation, contraction and pressure changes is for the purpose of obtaining and regulating this permeability. The facts bearing on this phase of capillary function are extremely meager.

A knowledge of physiologic function is often added to materially by a study of pathologic changes and their effects. Of special importance in connection with capillary function is the study of the early stages of acute inflammation. It may be assumed that inflammation occurs only when some injurious agent introduced from without or formed from damaged cells comes in contact with an area of tissue. As MacCallum ${ }^{2}$ points out, the first evidence of inflammation consists in a widening of the visable capillaries and the opening of new, hitherto unseen capillary areas. At this time there is an increase in the rate of blood flow. These changes are the ones usually described as following the application of any chemical irritant. Very shortly, however, the rate of flow becomes slower, with the red cells occupying the middle of the capillary channel, and plasma and leukocytes occupying the marginal borders. Obviously this change is due to some alteration

\footnotetext{
* From the University and Bellevue Hospital Medical College.

1. Hooker: Physiol. Rev. 1:112, 1921.

2. MacCallum: Textbook of Pathology, Ed. 2, p. 136.
} 
in the capillary structure. Further, and probably from the beginning, a change in permeability occurs, which is demonstrable through the passage of fluid and leukocytes into the tissue.

The nature of the capillary change in inflammation is not clear. Klemensiewiez ${ }^{3}$ believes that there is a paralysis of the capillary walls and supports this by showing that they fail to respond to nervous or direct stimulation. A simple paralysis would fail to explain the whole process, and Samuel, ${ }^{4}$ who holds that the tissue lesions are always secondary to the vascular ones, concludes that there is primarily some unexplained but specific effect on the capillary walls. In accepting this rather vague hypothesis it is evident that the specific effect may be induced by a vast number of chemical substances whose only common character is that they are irritants. This group produces its effect when applied locally, the effect comes on promptly, and the extent of injury is proportional to the concentration of the irritant and the length of time it remains in contact with the tissue acted on. It must be admitted that although the capillary walls may be especially sensitive to the irritant, other tissues may similarly be damaged by direct action. But the fact seems clear that in acute inflammation there is primarily an injury to the capillary endothelium which manifests itself not only in change in caliber but in abnormal permeability as well.

There is another group of substances which act similarily on the capillaries when brought to them by the circulation and which, in very minute doses, produce wide spread effects. The action on the capillaries appears to be as selective as that of strychnin on the nervous system, or digitalis on the heart. These substances, are classed pharmacologically as capillary poisons. The term has a wide application. It includes those substances which cause a widening or paralysis of the capillaries without inducing visible structural change. It includes also those which produce changes in caliber, accompanied or followed by structural changes. As a representative of the first group, histamin may be mentioned. This induces a wide spread but transient capillary dilatation. $^{5}$ The second group is made up of substances of such varying chemical nature as uranium, cantharidin, emetin, diptheria toxin, sepsin and gold. While many of these are irritants, this property does not explain the wide spread effects produced by minute dosage, unless one conceives a highly specific and selective irritant action. This group in its initial action produces a dilatation of capillaries. Very shortly, however, a structural damage may become apparent and constitute the main feature. While the action appears at times to be localized,

3. Klemensiewiez: Entzündung, Jena, 1908.

4. Samuel: Virchows Arch. f. path. Anat. 121:273-396, 1890.

5. Dale and Richards: J. Physiol. 52:110, 1918. 
this, as a matter of fact, is not strictly the case, for they may act on capillaries generally. They tend, however, to have a more intense effect on one organ or tissue than on others, this effect overshadowing other, less intense ones. Thus uranium and cantharidin are often considered purely as kidney poisons and emetin as an intestinal poison. The explanation of the evident selectivity of action lies in the excretion of the poison or other factors which determine its concentration. Thus, if given by injection into the jugular vein, the lung capillaries may be the ones chiefly affected, because the poison comes to them in greatest concentration. Those excreted by the kidney produce their greatest effects here, those excreted by the intestine, on that organ. However, if these localized effects are not severe enough to cause early death, changes elsewhere can usually be detected.

Some of these poisons act much more generally than others and this is especially the case with gold salts, sepsin and diptheria toxin. If the mesentery of a frog is observed under a low power microscope, in from one half to one minute after the injection of gold-sodium chlorid, ${ }^{6}$ new capillary fields suddenly appear, the rate of flow is rapid and then quickly slows. In mammals the veins become strongly distended and hemorrhages are numerous in the liver, spleen, kidney and intestine. With diptheria toxin ${ }^{7}$ there is marked injection of the small vessels and wide spread destruction of capillaries with outpouring of blood. Sepsin ${ }^{8}$ also causes wide spread hyperemia and hemorrhages. Similar effects may be obtained with emetin, ${ }^{9}$ but these are confined to the stomach and intestine, as is the case with colchicine. Such extreme effects as these must, of course, abolish entirely the function of the capillaries involved.

If it is true that these poisons, when their action is well developed, produce structural injury and impaired permeability of the capillaries, it follows that definite effects from these changes should be obtainable and measurable. Among these effects would be departures from the normal metabolism of tissues supplied by the damaged capillaries, departures proportional to the change in permeability. If the capillary changes come on gradually, are not too intense in their development and are of sufficient duration, it is reasonable to believe that the resulting inadequate nutrition of tissue would result in connective tissue growth and replacement, and in various types and stages of tissue degeneration. This, of course, is not a new idea. It was expressed in 1872 by Gull and Sutton, ${ }^{10}$ who believed that a general morbid state, which they

6. Heubner: Ach. f. exper. Path. u. Pharmakol. 56:370, 1907.

7. Wallace and Myers: J. Pharmacol. \& Exper. Therap. 6:601, 1915.

8. Faust: Arch. f. exper. Path. u. Pharmakol. 51:248, 1904.

9. Wallace and Pellini: Am. J. M. Sc. 152:325, 1916.

10. Gull and Sutton: Med. Chir. Tr. 55:273, 1872. 
termed arteriocapillary fibrosis, may exist, characterized hy hyalin fibroid formation in the walls of the minute arteries and hyalin granular changes in the capillaries. This morbid state is attended with atrophy of adjacent tissues, and the contracted kidney of chronic Bright's disease is only a part of the process. This view has been recently again expressed and enlarged by Moschowitz. ${ }^{11}$

Now, while it is easy to surmise a causal relationship between capillary injury and tissue degeneration, it must be emphasized that this relationship, as far as human pathology is concerned, is as yet a theory and not a fact. In the first place, the existence of poisons which might be expected to cause the capillary damage is hypothetical. In the second place, as Oertel has stated, there is no proof at present that vascular changes are the forerunners of tissue degenerations, and not part of a damaging process which affects capillaries and tissue independently. In order to leave the realm of theory and enter that of fact, it will be necessary, first of all, to produce by means of a known vascular poison, capillary injury which will be followed by tissue degeneration, and to vary the latter according to the extent and duration of the former. Experimental work along this line has so far not been very successful. Most of it has been carried on with the purpose of producing chronic nephritis. Dickson ${ }^{12}$ has produced a chronic diffuse nephritis, with severe glomerular lesions, by means of uranium. In advanced cases, along with other changes, there was a thickening of the capillary walls. He believes, however, that the vascular damage is of slight importance. On the other hand Harvey, ${ }^{13}$ using parahydroxy phenylethylamin, induced chronic kidney lesions which he considers to be secondary to vascular changes in the renal artery and its branches.

There is one other aspect of the effects of capillary injury, namely, its relation to high blood pressure. The popular conception at present is that at least one type of high blood pressure, the so-called essential hypertension, has as its basic cause a narrowing or. obliteration of capillary fields. As is the case with tissue degeneration, this relationship is a very plausible one, but again, is a theoretical conception. The problem does not appear beyond solution, however, and we are at present engaged on it in the hope of eventually getting some positive results.

\section{EXPERIMENTAL WORK}

In the course of our work we have looked for capillary poisons which have a wide spread rather than a localized sphere of action. This separation is not as simple as it appears. It seemed to us,

11. Moschowitz: Arch. Int. Med. 26:259 (Aug.) 1920.

12. Dickson: Arch. Int. Med. 9:557 (Oct.) 1912.

13. Harvey: J. Path. \& Bacteriol. 16:95, 1911. 
however, that a poison which has a rather general effect on capillaries should bring about some measurable change in metabolism, for a lessened capillary permeability ought to lead to a decrease in cell nutriment, and, included with this, a decrease in oxygen. This latter state could readily induce a condition of suboxidation. The ordinary metabolism experiment throws no definite light on this question. There is an increase in nitrogen elimination and other changes, which admit of various interpretations. It seemed, however, that one distinctive type of metabolic derangement might occur, namely, acidosis, and accordingly we have carried out a series of experiments using a number of capillary poisons, and determining their effects on the alkali reserve of the blood. Our results have been definite enough to make it seem desirable to report them in some detail.

The experiments were performed on adult dogs in a good state of nutrition, which were picked indiscriminately from the available laboratory animals. The drug used was given by subcutaneous, intramuscular or intravenous injection. No restriction in diet was made, although in the longer experiments the animals refused food as time went on. Blood to be tested was withdrawn from the external jugular vein once a day or oftener. The blood was received directly without exposure to air, into a tube containing potassium oxalate and mineral oil. It was immediately centrifuged and the serum analyzed for alkali reserve by the Van Slyke method. ${ }^{14}$

We feel it unnecessary to quote all our experiments and are limiting our protocols in general to one typical experiment for each poison tested.

\section{PROTOCOLS OF EXPERIMENTS}

Uranium.-Dog, weight, 7.2 kilos; uranium nitrate, $0.021 \mathrm{gm}$., injected subcutaneously October 5. Alkali reserve before injection 57.7 volume, per cent. carbon dioxid. After injection, October 6, 53.8 per cent.; October 7, 36.8 per cent.; October 8. 29.5 per cent.; October 9. 25.3 per cent.; October 11, 31.5 per cent.; October 12, 23.3 per cent.; October 13, exitus.

The twenty-four hour urine output rose from 330 c. c. on October 6 to 1,084 c. c. October 10 . It then fell and was 115 c.c. October 12.

Cantharidin.-Dog, weight $12 \mathrm{~kg}$., cantharidin, dissolved in weak sodium hydrate solution, injected intravenously as follows: November $23,5 \mathrm{mg}$; November, $24,7.5 \mathrm{mg}$; November $25,26,27,10 \mathrm{mg}$. Alkali reserve, before injection 58 per cent.; after injection, November 24, 58.2 per cent.; November 25, 58.4 per cent.; November 26,10 a.m., 40.9 per cent., $4: 30$ p. m., 36.2 per cent.; November 27, 42.5 per cent.; November 28, 30.9 per cent. Animal killed November 28. There was no anuria during the experiment.

Diptheria Toxin.-Dog, weight, $12.1 \mathrm{~kg}$., diptheria toxin m. 1. d. $1 / 500$ c. c., (obtained from Dr. W. H. Park), 1.4 c.c. injected intramuscularly at 4:30 p. m. November 30. Alkali reserve before injection, 558 per cent. After injection, December 1, 9:30 a. m., 30.3 per cent.; 11:40 a. m., 18.1 per cent. Exitus, December 1 .

14. Van Slyke: J. Biol. Chem. 30:347, 1917. 
We have used in these experiments three capillary poisons, two of which, uranium ${ }^{15}$ and cantharidin, ${ }^{16}$ act especially on the kidney; the third, diptheria toxin, ${ }^{7}$ acting equally strongly on the intestinal and liver capillaries, and, in fact, on capillaries generally. The action of uranium and cantharidin is not limited to the kidney, however.

In order to determine to what extent the renal action was concerned in the acidosis, the following experiments were done. In a control animal a double nephrectomy was performed under ether anesthesia and the blood was analyzed during the four following days. In a second animal, a double nephrectomy was performed, and on the following day uranium was injected. We are indebted to Dr. W. H. Barber for the operative procedures. The results were as follows:

Nephrectomy.-Dog, weight, $15.8 \mathrm{~kg}$., double nephrectomy November 10. Alkali reserve, before operation, 57.7 per cent.; after operation, November 11, 49 per cent.; November 12, 60.2 per cent.; November 13, 61.1 per cent.; November 14, 69 per cent.

Nephrectomy and Uranium.-Dog, weight $11.25 \mathrm{~kg}$, double nephrectomy November 16 . November $17,33 \mathrm{mg}$. uranium nitrate by subcutaneous injection. Alkali reserve before nephrectomy, 50 per cent.; after nephrectomy, November 17, 52.3 per cent.; after uranium, November 18, 44 per cent.; November 19, 38.7 per cent.; November 20, 10 a. m., 33.1 per cent., $2: 30$ p. m., 30.7 per cent.

It is obvious from these experiments that removal of both kidneys is without influence on uranium acidosis, and that kidney damage is not an appreciable factor in this acidosis.

Emetin,-Dog, weight, $13.1 \mathrm{~kg}$., emetin hydrochlorid, 1 per cent. solution, injected subctutaneously, 2.5 c. c. daily, October 12,13 and 14 ; twice October 15 , 3 c. c. twice October 16 . Alkali reserve, before injection, 63.6 per cent.; after injection, October 12, 63.3 per cent.; October 13, 63.3 per cent.; October 14, 57.9 per cent.; October 15, 57.9 per cent.; October 16, 63.5 per cent.; October 17, 57.6 per cent. Exitus, October 18.

In another emetin experiment a dog was given $21 \mathrm{c}$. c. of a 1 per cent. solution during a period of four days. At the end of this time, under morphin and light ether anesthesia, blood was withdrawn from the jugular, portal and an intestinal vein at approximately the same time. The alkali reserve was 54.8 per cent., 54.8 per cent. and 53.9 per cent., respectively.

Podophyllotoxin.-Dog, weight, $11.8 \mathrm{~kg}$, podophyllotoxin, dissolved in weak alkali, injected intramuscularly as follows: October $28,20 \mathrm{mg}$; October 29, $30 \mathrm{mg}$.; October 30, $20 \mathrm{mg}$.; November 2, $20 \mathrm{mg}$; November 3, $30 \mathrm{mg}$. Alkali reserve, before injection, 57.8 per cent.; after injection, October 28, 58.6 per cent.; October 29, 57.5 per cent.; October 30, 539 per cent.; October 31, 58,6 per cent.; November 1, 55.7 per cent.; November 3, 53.8 per cent.; November 4, 53.9 per cent.; November 5, 44.5 per cent. Exitus, November 5, shortly after last blood examination.

15. McNider: J. M. Res. 31:79, 1912.

16. Lyon: J. Path. \& Bacteriol. 9:444, 1904. 
Emetin $^{9}$ and podophyllotoxin ${ }^{17}$ act selectively on the intestinal capillaries, and in the experiments just noted necropsy showed the presence of the characteristic intestinal lesions. There was also congestion of the kidney, but the renal damage was not severe, as the urine showed only faint traces of albumin and no striking diminution in quantity. In the second emetin experiment we had expected to find the alkali reserve in the blood from the intestinal and portal veins lower than in that from the jugular vein. In this we were disappointed and have to conclude that marked damage to the intestinal capillaries fails to bring about an appreciable acidosis.

Arsenic.-Dog, weight, $16.7 \mathrm{~kg}$, arsenious acid given subcutaneously in daily dosage of $30 \mathrm{mg}$., November $18,19,20$ and 21 ; intravenously November 22,30 mg.; November 23, $30 \mathrm{mg}$.; November 24, $50 \mathrm{mg}$.; November 25, $70 \mathrm{mg}$.; November $26,70 \mathrm{mg}$.; November $28,80 \mathrm{mg}$. Alkali reserve, before injection, 54 per cent.; after injection, November 19, 54.9 per cent.; November 20, 55.9 per cent.; November 21, 55.7 per cent.; November 22, 55.6 per cent.; November 23, 55.6 per cent.; November 24, 55.6 per cent.; November 25, 57.4 per cent.; November 26, 46.2 per cent.; November $27,45.3$ per cent. Exitus, November 28.

In this experiment, the arsenic acted chiefly on the intestinal tract. There was also well marked congestion of the liver and kidneys, and a beginning fatty change in the liver. ${ }^{18}$ The acidosis was not marked, and this we attribute to an absence of wide spread capillary damage. In another arsenic experiment we obtained the following result :

Arsenic.-Dog, weight $13.4 \mathrm{~kg}$, $60 \mathrm{mg}$. arsenious acid injected intravenously at $11: 15$ a. m. Alkali reserve, before injection, 50 per cent.; at 2:45 p. m., 30.4 per cent. Exitus, $3: 15$ p. m.

Hydrazin.-Dog, weight, $10.6 \mathrm{~kg}$., hydrazin sulphate injected subcutaneously $100 \mathrm{mg}$. daily, November 8,9 and $10 ; 200 \mathrm{mg}$. daily November 11, 12, 13 and $14 ; 400 \mathrm{mg}$. November 15 . Alkali reserve before injection 53.8 per cent.; after injection, November 9, 51 per cent.; November 10,53.9 per cent.; November 11, 55.7 per cent.; November 12, 62.6 per cent.; November 13, 67.2 per cent.; November 14, 67.1 per cent.; November 15, 65.3 per cent. Exitus, November 16.

This substance is not classed as a capillary poison. It has a highly selective action on liver tissues, however, producing marked destruction and fatty infiltration of liver cells. ${ }^{19}$ In another hydrazin experiment comparable to the second arsenic and the diptheria experiments in regard to time, the following result was obtained:

Dog, weight, $618 \mathrm{~kg}$., $600 \mathrm{mg}$. hydrazin sulphate injected subcutaneously at 3:45 p.m. November 5. Alkali reserve before injection, 52 per cent.; after injection, 11 a. m., November 6,72 per cent. Exitus, 1 p. m., November 6.

With this marked damage to the liver, we find, therefore, not an acidosis, but the reverse.

17. Neuberger: Arch. f. exper. Path. u. Pharmakol. 28:32, 1890 .

18. Boehm: Arch. f. exper. path. u. Pharmakol. 2:89, 1874.

19. Underhill and Klein: J. Biol. Chem. 4:165, 1908 
As the above described experiments show that neither kidney, intestine or liver injury is responsible for the acidosis we have obtained, we carried out the following transfusion experiment to determine whether the muscle tissue is concerned in the process.

January 25 a dog was given 1.75 c. c. diptheria toxin injected intramuscularly. The alkali reserve at $11 \mathrm{a}$. $\mathrm{m}$. January 26 was 35 per cent. At $11: 30 \mathrm{a}$. m. the femoral artery and vein of this dog were connected with the femoral artery and vein of a normal dog, the latter, therefore, supplying the circulation to the poisoned leg. The necessary operative procedures were carried out under morphin anesthesia, supplemented with a small quantity of cocain. Hirudin was used to prevent clotting of blood The transfusion was maintained for over an hour. The alkali reserve of the normal dog before the experiment began was 55.8 per cent. The figures obtained after 45 minutes of transfusion were, jugular blood, normal dog, 55.4 per cent.; diptheria toxin dog, 31.5 per cent.; femoral blood from poisoned leg, 42.6 per cent.

Using the bloods taken from these two animals just before the transfusion, we have estimated the hemoglobin and blood cells, and made the ordinary chemical analysis.

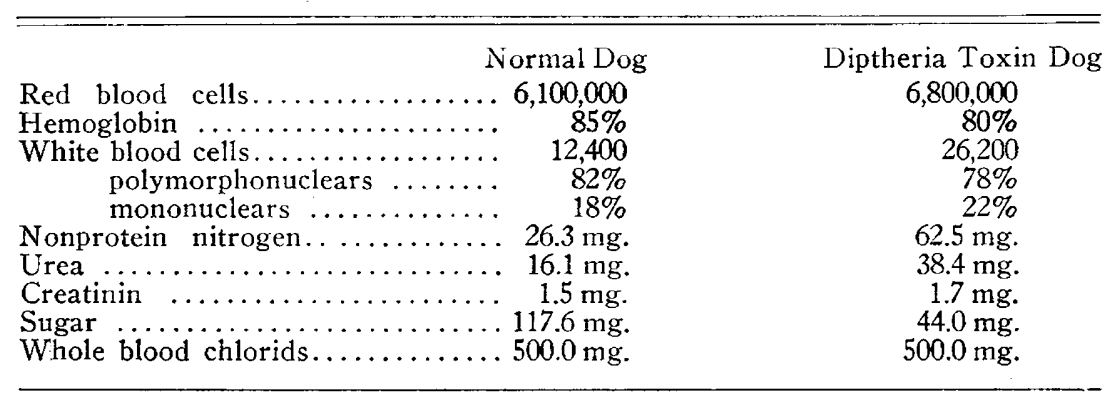

A comparison of the red blood cell count and hemoglobin shows no difference in the concentration of the blood in the two animals. The diptheria toxin animal shows a leukocytosis and in addition the altered blood chemistry which is to be expected, since the toxin acts on the kidney as well as other capillaries.

An experiment of this sort is naturally open to some criticism from the technical factors involved, but the results show that muscle is one of the tissues involved in the production of acidosis.

We have assumed that a suboxidation due to impaired capillary permeability is the cause of the acidosis from capillary poisons. It seemed to us that this assumption would be strengthened by obtaining acidosis through suboxidation induced by other than capillary poisons. The following experiments bear on this point.

Sodium Nitrite.-Dog, weight, $11.6 \mathrm{~kg}$., received by subcutaneous injection $0.1 \mathrm{gm}$. sodium nitrite January 10 at hourly intervals from $11: 45 \mathrm{a}$. m. to $3: 45$ p. m., in all $0.5 \mathrm{gm}$. Alkali reserve before injections, 51.4 per cent., at $4 \mathrm{p} . \mathrm{m}$. 58 per cent. The blood spectroscopically showed no methemoglobin. On the following day, the animal received $0.2 \mathrm{gm}$. sodium nitrite, at intervals, from 
$10: 45$ a. m. to $12: 45$ p. m., $0.8 \mathrm{gm}$. in all. Alkali reserve before injection, 56.1 per cent., at $1: 10$ p. m. 49 per cent., at $1: 50$ p. m., 29 per cent. At 1 p. m. blood showed methemoglobin.

It appears from this experiment that a lowering of blood pressure which must have been induced by the nitrite on the first day of the experiment was not productive of acidosis. This appeared only on the second day when the formation of methemoglobin occurred. Methemoglobin, of course, means cellular asphyxia.

Potassium Cyanid.-Dog, weight, $11.3 \mathrm{~kg}$., received $34 \mathrm{mg}$. potassium cyanid January 12 at 11 a. m.; $22 \mathrm{mg}$. at $11: 30$ a. m.; $50 \mathrm{mg}$. at 12 noon. No characteristic symptoms, except vomiting and a slight ataxia occurred until 12:25 p. m., when marked dyspnea and muscle twitchings appeared. Alkali reserve before injections, 53.8 per cent.; after injections, 12 noon, 27.4 per cent.; $12: 25$ p. m., 12.2 per cent. Exitus during night.

Potassium cyanid has been studied quite extensively from the standpoint of its effect on metabolism. ${ }^{20}$ It acts in some unexplained way on tissues, preventing their using available oxygen. It may be noted in this experiment that the acidosis was very definite before any convulsive movements had set in.

We have one other experiment to report. In most of our experiments, as poisoning progressed, there developed a condition of muscular weakness, prostration, narcosis and lessening of the respiratory function. We have reproduced this condition by administering large quantities of morphin. The results are as follows.

Morphin.-Dog, weight $10.3 \mathrm{~kg}$, normal respiration, 20 per minute. December 1, a total of $190 \mathrm{mg}$. morphin sulphate given by subcutaneous injection at half hour intervals from 10:45 a. m. to 5:10 p. m. The respiration fell to 11 per minute, slightly labored, with moderate narcosis. Alkali reserve before morphin, 64 per cent.; after morphin, at 4:20 p. m., 61.5 per cent. On the following day, morphine was again given, at frequent intervals, from 9:55 a. m. to $3 \mathrm{p}$. m. In all $2 \mathrm{gm}$. morphin were injected. At $3: 30 \mathrm{p} . \mathrm{m}$. respiration was 10 per minute and shallow, moderate narcosis, animal being unable to stand. At this time the alkali was 64 per cent. Death occurred during the night.

The administration of large amounts of morphin, therefore, with the production of narcosis and respiratory depression, does not cause acidosis. Gauss ${ }^{21}$ has recently shown that an actual increase in alkali reserve may occur.

\section{DISCUSSION}

In looking over these experiments we find that those poisons which produce a wide spread capillary damage, namely uranium, cantharidin and diphtheria toxin, cause a definite acidosis. The. first two of these

20. Richards and Wallace: J. Biol. Chem. 4:179. 1908.

21. Guass: J. Pharmacol. \& Exper. Therap. 16:475, 1921. 
act most strongly on the kidney; but the fact that uranium is equally effective in causing acidosis in nephrectomized animals speaks against renal damage being an essential factor.

Emetin and podophyllotoxin produce very destructive effects along the intestinal tract, and yet neither causes an appreciable acidosis. Again, hydrazin is a powerful liver poison, but its administration in fatal dose is not followed by acidosis.

The transfusion experiment described, in which a diminished alkali reserve is seen in venous blood from a diptheria toxin poisoned extremity, receiving normal arterial blood, offers a clue to the place of origin of the acidosis, and we believe the abnormal metabolism, of which acidosis is one of the evidences, occurs chiefly in muscle tissue.

The explanation of the abnormal metabolism lies, we believe, in a diminished permeability of the damaged capillary wall, the diminution in the oxygen reaching the tissue cell resulting in a state of suboxidation. This belief is strengthened by the experiments with sodium nitrite and potassium cyanid, which show that a failure of the blood to give up oxygen, due to the formation of methemoglobin, or of the tissue to utilize available oxygen, will also cause acidosis.

Finally, in application of our experimental facts, we believe that many cases of clinical acidosis may be explained by capillary injury. An acidosis, for example, may occur in many, if not all of the acute infectious diseases. Thus it has been observed in pneumonia, ${ }^{22}$ in diptheria, ${ }^{23}$ in scarlet fever ${ }^{24}$ and in influenza. ${ }^{25}$ While little is known concerning the toxins in most of these infections, in one at least, diptheria, the toxin is known and is the poison which, in our experiments, produced the most striking degree of acidosis obtained.

Among other clinical cases which were accompanied by acidosis we find nephritis, ${ }^{26}$ and cardiac disease. ${ }^{27}$ These conditions are considered by many to be part of a general vascular disease, the arteriocapillary fibrosis of Gull and Sutton, and looking at them from this standpoint, a relationship between the acidosis and capillary injury can easily be assumed.

\section{SUMMARY}

1. Those poisons which have a wide spread action on capillaries, produce a marked degree of acidosis. Among these are diptheria toxin, uranium and cantharidin.

22. Palmer: J. Exper. M. 26:495, 1917.

23. Peters: Brit. M. J. 1:10, 1918.

24. Thomas: Ibid. 1:274, 1919.

25. Hachen and Isaacs: J. A. M. A. 75:1624 (Dec. 11) 1920.

26. Peabody: Arch. Int. Med. 16:955 (Dec.) 1915.

27. Peabody: Ibid. 14:23 (July) 1914. 
2. Renal injury is not an essential factor in the acidosis, since double nephrectomy fails to produce acidosis, and this condition can be brought about in nephrectomized animals.

3. Those poisons which act selectively on the intestinal capillaries fail to produce acidosis, or if so, produce it to a minor degree.

4. Marked injury to liver tissue does not cause acidosis.

5. Evidence is submitted which points to muscle tissue as being one of the seats of acid formation.

6. The assumption is made that the cause of the acidosis is a condition of suboxidation in the tissues, and experiments are reported showing that suboxidation produced by other means than capillary poisoning also causes acidosis.

7. Capillary poisoning is suggested as an explanation of acidosis occurring in acute infectious diseases and some other clinical conditions. 